\title{
Influencia de la concentración de aceite esencial de orégano (Origanum vulgare L.) en el tiempo de vida en anaquel del aceite de oliva (Olea europea) extravirgen
}

\author{
Diana Nolazco Cama*, Lena Téllez Monzón**, Karina Ccapa Ramírez*** \\ Universidad Nacional Agraria La Molina. Lima, Perú.
}

Recibido: 26 de junio de 2015 / Aprobado: 27 de julio de 2015

RESUmEN: Se evaluó la influencia de dos concentraciones de aceite esencial de orégano: $0,1 \%$ y $0,3 \%$, aplicados a un aceite de oliva extravirgen con un $\%$ de acidez y de índice de peróxido inicial de 0,168 y 9,5 miliequivalente de peróxido/kg, respectivamente. Para la estimación de la vida en anaquel, las muestras de aceite de oliva fueron almacenadas a tres temperaturas: $50{ }^{\circ} \mathrm{C}, 60^{\circ} \mathrm{C}$ y $70{ }^{\circ} \mathrm{C}$, y se evaluó los cambios producidos en el \% de ácidos grasos libres e índice de peróxido como factores de calidad durante el almacenaje. Los análisis demostraron que el aceite de oliva se oxida en menor proporción cuando la concentración del aceite esencial es mayor.

Palabras clave: aceite de orégano / aceite de oliva extravirgen / ácidos grasos libres / peróxidos

\section{Influence of the Concentration of Essential Oil of Oregano \\ (Origanum vulgare $L$.) in the Shelf Life Time of Virgin Olive}

\section{(Olea europea) Oil}

ABstract: The influence of two concentrations of essential oil of oregano: $0,1 \%$ and $0,3 \%$, applied to extra virgin olive oil with an acidity $\%$ and initial rate of $0,168 \%$ peroxide and 9,5 milliequivalent of peroxide was evaluated / kg, respectively. For estimating the shelf life, samples of olive oil were stored at three temperatures: $50{ }^{\circ} \mathrm{C}, 60^{\circ} \mathrm{C}$ and $70{ }^{\circ} \mathrm{C}$, evaluating changes in the \% FFA and peroxide as quality factors for the storage. Analysis showed that the olive oil is oxidized to a lesser extent when the concentration of essential oil is greater.

Key words: oregano oil / extra virgin olive oil / free fatty acids $/$ peroxides

Correos electrónicos: *denolazco@lamolina.edu.pe, **ltellez@lamolina.edu.pe, ***karinabcr@gmail.com 


\section{INTRODUCCIÓN}

Los aceites sufren pronunciados cambios oxidativos, especialmente cuando son expuestos a altas temperaturas, a la luz y, específicamente, a la presencia de oxígeno durante el almacenamiento. En estudios anteriores se sometieron aceites de oliva virgen a temperaturas de almacenamiento altas, y se encontró que el porcentaje de ácidos grasos libres y el índice de peróxido eran los factores de calidad que variaban con el tiempo, incrementándose en ambos casos y siendo determinantes en la estimación del tiempo de vida útil de los aceites de oliva.

El aceite de oliva está formado básicamente de triacilgliceroles (98 \% - 99 \%). La composición característica básica de los ácidos grasos que forman los triacilgliceroles del aceite de oliva muestra claramente el predominio de ácido oleico, entre otros (tabla 1). La oxidación de los ácidos grasos producen dienos conjugados que se oxidan formando hidroperóxidos.

Tabla 1

Composición básica de los ácidos grasos mayoritarios del aceite de oliva

\begin{tabular}{lc}
\hline Ácidos grasos libres & Límites (\%) \\
\hline Palmítico & $7,5-20,0$ \\
Palmitoléico & $0,3-3,5$ \\
Esteárico & $0,5-5,0$ \\
Oleico & $55,0-83,0$ \\
Linoleico & $3,5-21,0$ \\
Linolénico & $\leq 1,0$ \\
\hline
\end{tabular}

Fuente: Soler (2009)

Estos cambios oxidativos reducen su calidad nutricional y una forma de evitarlos es mediante la adición de algunos antioxidantes comúnmente sintéticos, a pesar de que el aceite de oliva presenta una baja concentración de compuestos fenólicos con importancia destacable de efecto antioxidante, no es suficiente para resistir la influencia de algunos factores oxidantes (luz, oxígeno y temperatura). Después de la evaluación de las cinéticas de oxidación, el aceite de oliva es más 
resistente a la oxidación por su composición baja en ácidos grasos insaturados y la presencia de tocoferoles y fenoles; sin embargo, a pesar de ello no está libre de la oxidación.

La seguridad en el uso de los antioxidantes sintéticos ha sido cuestionada en la actualidad, por sus efectos negativos sobre la salud. En tanto, es ampliamente conocido que los aceites esenciales son usados como antioxidantes; el orégano contiene timol, carvacrol y ácidos fenólicos, a los cuales se les atribuye su actividad antioxidante y antimicrobiana.

En el aceite esencial de orégano, el timol y el carvacrol (figura 1) son moléculas isoméricas que pueden frenar las reacciones de iniciación de la cadena durante la oxidación de los triglicéridos de un aceite vegetal. Además, el carvacrol puede participar bloqueando la reacción de propagación de la cadena, pero el timol no.

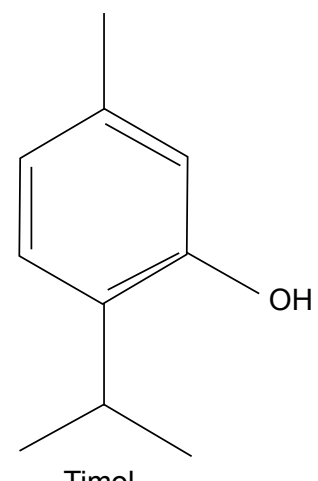

Timol

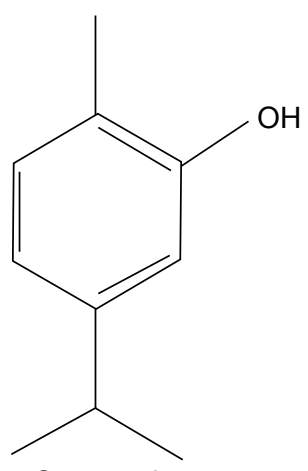

Carvacrol

Figura 1. Moléculas de timol y carvacrol Fuente: Asencio (2013)

Incluso, el aceite esencial de orégano estudiado sensorialmente en muestras de aceite de oliva indican que aumentan en general la intensidad del sabor amargo y picante del aceite, siendo aceptable en cuanto a los atributos de color, olor y sabor. Un aceite de oliva preparado con la adición de aceite esencial de orégano podría no solo proteger el aceite de oliva de la oxidación de lípidos, actuando como un antioxidante natural, sino también preservaría su sabor, aroma y propiedades nutricionales beneficiosas para la salud, e incluso podría aportar una combinación de sabor que podría resultar más agradable para algunos consumidores. 
Las pruebas aceleradas se han utilizado ampliamente para observar la estabilidad de los aceites vegetales, con el fin de predecir con un cierto margen de incertidumbre, la estabilidad o pérdida de calidad de un alimento. $\mathrm{Al}$ aumentar la temperatura de almacenamiento el nivel de oxidación crece, de tal modo que la totalidad de la prueba se puede realizar en un lapso más corto. La velocidad de las reacciones que se producen permite estimar la vida útil del alimento en función de las características de calidad evaluadas.

La pérdida de calidad en los alimentos puede ser representada por una ecuación matemática de la siguiente forma:

$$
\mathrm{dA} / \mathrm{d} \theta=\mathrm{kA}^{\mathrm{n}}
$$

Las reacciones en los alimentos pueden ser de orden cero, primer orden, segundo orden u otros. La ecuación matemática en cada caso es:

Reacciones de orden cero:

$$
\mathrm{A}=\mathrm{A}_{\mathrm{o}}-\mathrm{K} \theta_{\mathrm{s}}
$$

Reacciones de primer orden:

$$
\operatorname{Ln} A=\operatorname{Ln} A_{o}+K \theta_{s}
$$

Donde:

$\mathrm{A}=$ Valor de la característica de calidad en el tiempo $\theta$

$\mathrm{A}_{\mathrm{o}}=$ Valor inicial de la característica de calidad

$\mathrm{K}=$ Constante de velocidad de reacción

$\theta_{\mathrm{s}}=$ Vida en anaquel en días, meses, años, etc.

En los alimentos, las reacciones que comúnmente siguen una orden de reacción cero son: las degradaciones enzimáticas, los pardeamientos no enzimáticos, la oxidación de lípidos; mientras que algunos ejemplos de reacciones de primer orden son: rancidez de aceites o vegetales secos, crecimiento microbiano, producción microbiana de sabores indeseables y pérdida de vitaminas. Existen muy pocos datos para describir la degradación de alimentos por otras órdenes.

\section{PARTE EXPERIMENTAL}

\subsection{Lugar de ejecución}

La investigación fue realizada en los laboratorios del Departamento Académico de Química de la Facultad de Ciencias, y en los laboratorios 
de Fisicoquímica de la Facultad de Industrias Alimentarias de la Universidad Nacional Agraria La Molina.

\subsection{Materiales}

\subsubsection{Muestras}

Para la investigación se utilizó un aceite de oliva extravirgen, con una acidez libre de $0,168 \%$ y un nivel de peróxidos de 9,5 miliequivalentes/ $\mathrm{kg}$, envasados en botellas de vidrio ámbar. El aceite esencial de orégano fue extraído mediante arrastre por vapor con un porcentaje de acidez de 1,68 , envasado en frascos oscuros y en congelación hasta su aplicación.

\subsubsection{Reactivos}

- Hidróxido de sodio $0.1 \mathrm{~N}$

- Fenolftaleína

- Acético: cloroformo (3:2)

- Solución saturada de yoduro de potasio

- Tiosulfato de sodio $0.01 \mathrm{~N}$

- Almidón al $1 \%$

\subsection{Metodología}

\subsubsection{Aplicación del aceite esencial en el aceite de oliva extravirgen}

$\mathrm{El}$ aceite esencial de orégano fue aplicado en el aceite de oliva en las dosis de 0,1 y $0,3 \%$, después de la homogeneización estas fueron envasadas en frascos de vidrio oscuros de $50 \mathrm{ml}$ cada uno. El mismo procedimiento se realizó para cada temperatura de almacenamiento $\left(50^{\circ} \mathrm{C}, 60^{\circ} \mathrm{C}\right.$ y $\left.70^{\circ} \mathrm{C}\right)$.

\subsubsection{Almacenamiento de las muestras}

Las muestras se almacenaron en cámaras a $50^{\circ} \mathrm{C}, 60^{\circ} \mathrm{C}$ y $70^{\circ} \mathrm{C}$. Los análisis del porcentaje de ácidos grasos libres e índice de peróxido se realizaron durante 24 días. Cada análisis se realizó por triplicado. 


\subsubsection{Determinación de la vida en anaquel}

Con los datos del porcentaje de acidez e índice de peróxido durante el almacenamiento se realizó un análisis de regresión que mejor se ajuste a los datos, obteniendo la velocidad de reacción $(\mathrm{k})$ para cada factor de calidad, y extrapolándose a una temperatura de $20{ }^{\circ} \mathrm{C}$ (temperatura ambiente). Finalmente, para obtener el tiempo de vida en anaquel $(\theta \mathrm{s})$ se utilizó las siguientes ecuaciones en función al porcentaje de ácidos grasos libres y el índice de peróxido:

$$
\begin{aligned}
& \% \mathrm{AGL}=\% \mathrm{AGLo}+\mathrm{K} \theta \mathrm{s} \\
& \mathrm{IP}=\mathrm{IPo}+\mathrm{K} \theta \mathrm{s}
\end{aligned}
$$

Los límites de calidad para los aceites de oliva extravirgen considerados fueron: $0,8 \%$ de ácidos grasos libres y 20 miliequivalentes de peróxido/kg, establecidos por el Consejo Oleícola Internacional.

\section{RESULTADOS Y DISCUSIÓN}

\subsection{Almacenamiento de las muestras}

Las figuras 2, 3 y 4 muestran la variación del porcentaje de los ácidos grasos libres determinados durante el almacenaje del aceite de oliva extravirgen a $50{ }^{\circ} \mathrm{C}, 60^{\circ} \mathrm{C}$ y $70{ }^{\circ} \mathrm{C}$, respectivamente, a las dosis de 0,1 y $0,3 \%$ de aceite esencial de orégano. Se observó que la acidez presenta un incremento con el tiempo en todas las muestras, debido a que los aceites con predominio de ácidos grasos insaturados son susceptibles a la hidrólisis química o enzimática, y es acelerada por las altas temperaturas. Es conocido que la temperatura es un factor tan primordial que afecta intensamente a las velocidades de degradación, y en términos generales la calidad del aceite disminuye al aumentar la temperatura en el transcurso del tiempo.

Según los resultados obtenidos, la variación en el porcentaje de ácidos grasos libres fue menor en aquellas muestras de aceite que contenían mayor cantidad de aceite esencial de orégano en todas las temperaturas de almacenamiento; esta respuesta puede atribuirse a la capacidad antioxidante de los aceites esenciales, conociendo que dentro de los componentes con estas propiedades se encuentra el timol y carvacrol, antioxidantes que se caracterizan por ceder hidrógeno a partir de los grupos fenólicos, interrumpiendo así la propagación de 
la oxidación a través de la cadena de radicales libres. De esta manera se forman compuestos estables que no pueden iniciar o propagar la oxidación lipídica. El aceite esencial de orégano tiene actividad antirradicalaria y esta propiedad se le atribuye a los mono-fenoles carvacrol y timol, principales quimiotipos, cada uno con enzimas específicas que dirigen su biosíntesis.

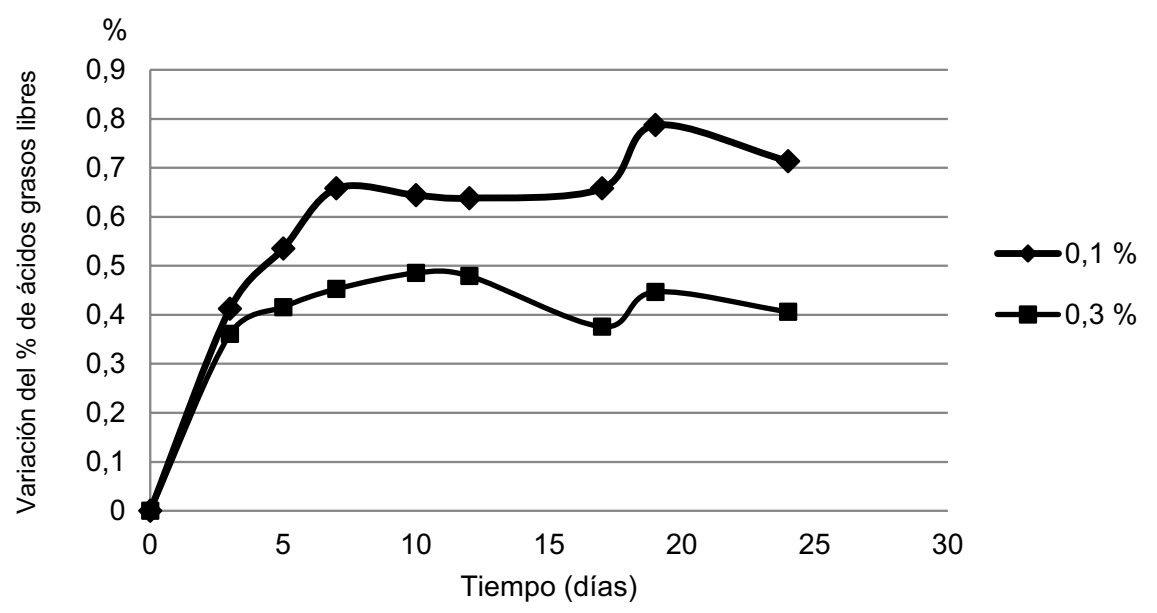

Figura 2. Variación del $\%$ de ácidos grasos libres a $50{ }^{\circ} \mathrm{C}$ con $0,1 \%$ y $0,3 \%$ de aceite esencial de orégano

Elaboración propia

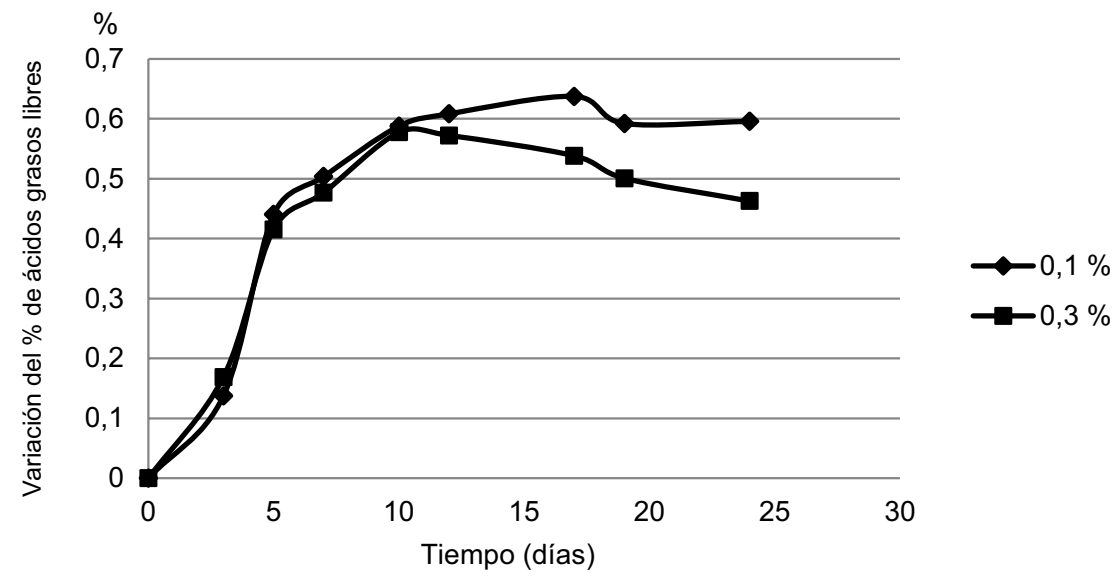

Figura 3. Variación del \% de ácidos grasos libres a $60{ }^{\circ} \mathrm{C}$ con $0,1 \%$ y $0,3 \%$ de aceite esencial de orégano

Elaboración propia 


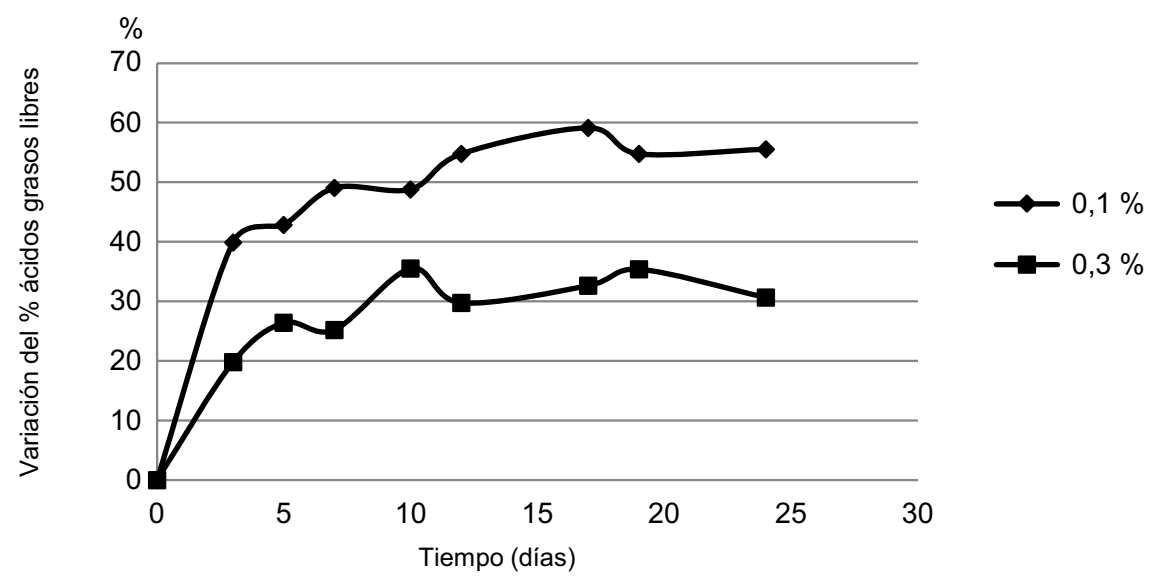

Figura 4. Variación del \% de ácidos grasos libres a $70{ }^{\circ} \mathrm{C}$ con $0,1 \%$ y $0,3 \%$ de aceite esencial de orégano

\section{Elaboración propia}

En la tabla 2 se presenta los porcentajes de variación de la acidez para las aplicaciones con aceite esencial de orégano durante el almacenamiento. La menor variación con respecto al valor inicial de acidez se presentó en los aceites de oliva que contenían una dosis de 0,3\% de aceite esencial de orégano. Se observó que a $70{ }^{\circ} \mathrm{C}$ la variación porcentual fue de $56 \%$ y $31 \%$ con la aplicación de $0,1 \%$ y 0,3 \% de aceite esencial, respectivamente.

Respecto al índice de peróxido, en las figuras 5, 6 y 7 se muestran un incremento al inicio del almacenamiento y un descenso al final, siendo este comportamiento o curva con menor incidencia en las muestras que contenían $0,3 \%$ de aceite esencial. El incremento de peróxidos en el tiempo es indicativo de la proporción de los primeros productos de la oxidación. La curva que se observa durante el almacenamiento es debido a que los peróxidos están sujetos a reacciones secundarias de degradación, alcanzando una concentración máxima que después disminuye debido a su descomposición. La capacidad antioxidante del aceite esencial de orégano aplicado en aceites de soya también presentó el mismo comportamiento respecto a un patrón que no contenía aceite esencial.

En el Origanum vulgare se ha comprobado el alto contenido en compuestos polifenólicos, que proveen una efectiva protección en todas las fases de la oxidación lipídica, esta actividad también es afectada por el tipo, composición y concentración del aceite esencial. 
Tabla 2

Variación del \% de acidez del aceite de oliva extravirgen

\begin{tabular}{ccc}
\hline $\begin{array}{c}\text { Temperatura } \\
\left({ }^{\circ} \mathrm{C}\right)\end{array}$ & $\begin{array}{r}\mathbf{0 , 1} \% \text { de aceite } \\
\text { esencial }\end{array}$ & $\begin{array}{c}\mathbf{0 , 3} \% \text { de aceite } \\
\text { esencial }\end{array}$ \\
\hline 50 & 71 & 41 \\
60 & 60 & 46 \\
70 & 56 & 31 \\
\hline
\end{tabular}

Elaboración propia

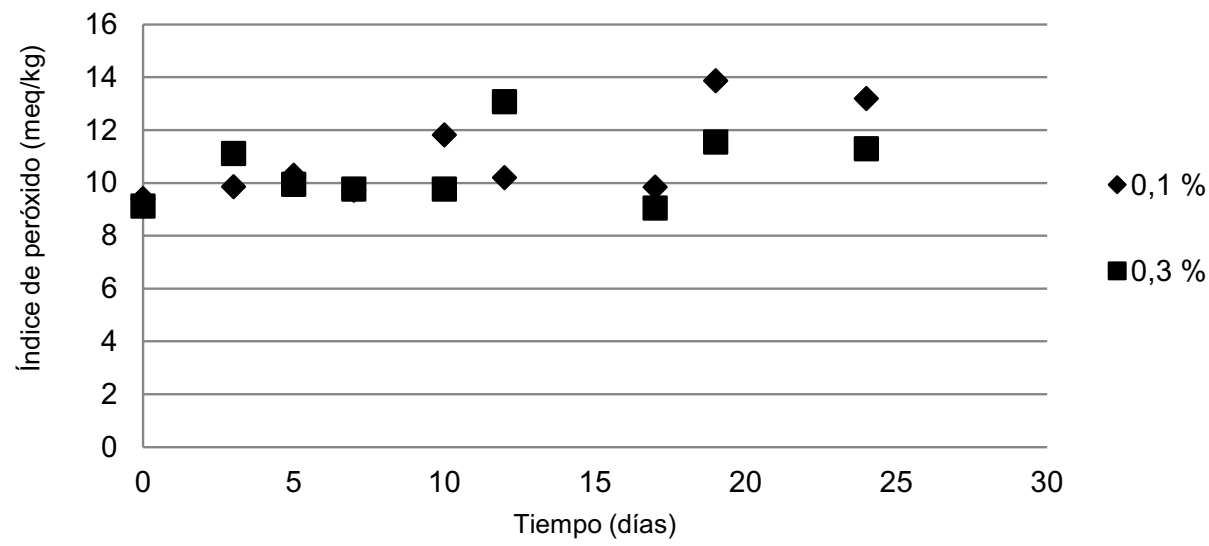

Figura 5. Índice de peróxido a $50{ }^{\circ} \mathrm{C}$ con $0,1 \%$ y $0,3 \%$ de aceite esencial de orégano Elaboración propia

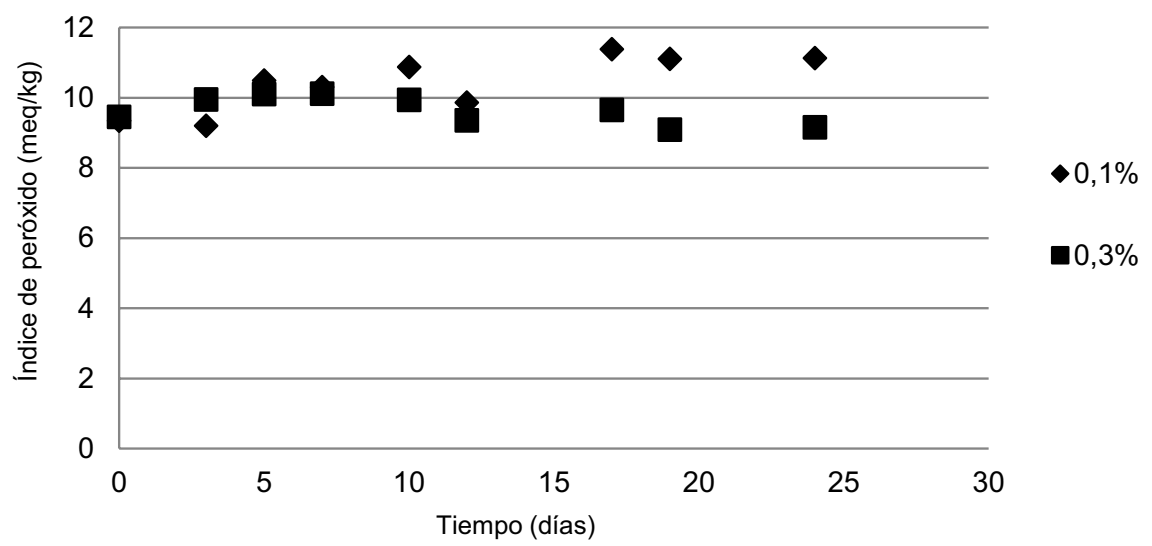

Figura 6. Índice de peróxido a $60{ }^{\circ} \mathrm{C}$ con $0,1 \%$ y $0,3 \%$ de aceite esencial de orégano Elaboración propia 


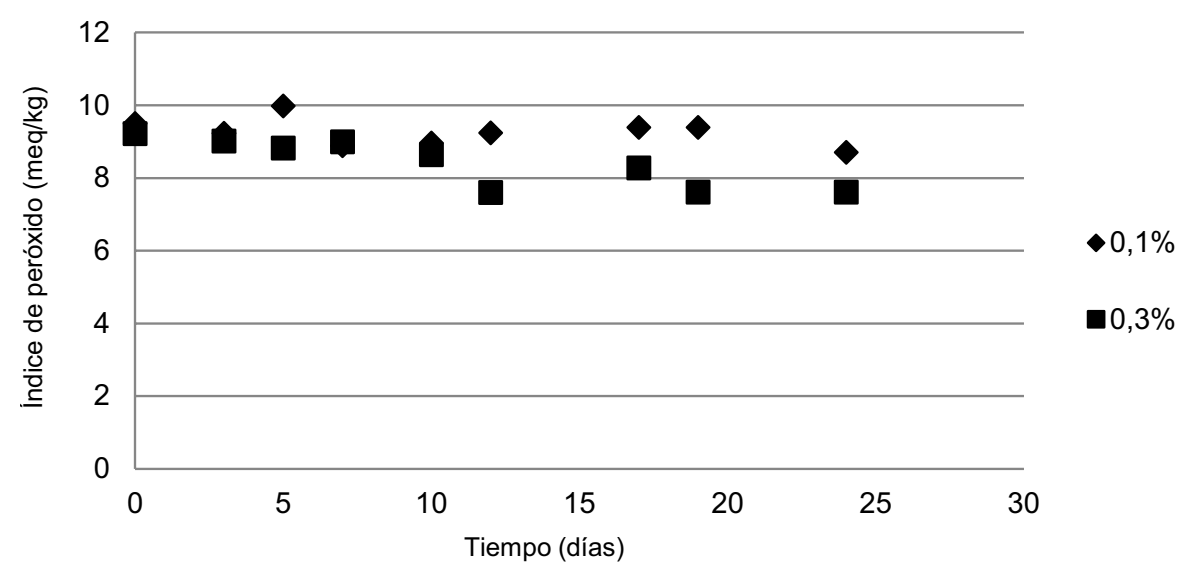

Figura 7 . Índice de peróxido a $70{ }^{\circ} \mathrm{C}$ con $0,1 \%$ y $0,3 \%$ de aceite esencial de orégano Elaboración propia

La estimación de la vida en anaquel del aceite de oliva extravirgen se determinó mediante regresión lineal de los valores del porcentaje de acidez e índice de peróxido para cada factor de calidad. La tabla 3 muestra los coeficientes de determinación lineal respecto a cada dosis de aceite esencial aplicado.

Tabla 3

Valores de $R^{2}$ para una dosis de 0,1\% y 0,3\% de aceite esencial

\begin{tabular}{lrrr}
\hline \multirow{2}{*}{ Factor de calidad } & \multicolumn{3}{c}{$\mathbf{R}^{\mathbf{2}} \mathbf{c o n} \mathbf{0 , 1} \mathbf{\%}$} \\
\cline { 2 - 4 } & $\mathbf{5 0}{ }^{\circ} \mathbf{C}$ & $\mathbf{6 0}{ }^{\circ} \mathbf{C}$ & $\mathbf{7 0}^{\circ} \mathbf{C}$ \\
\hline Ácidos grasos libres (\%) & 0,9524 & 0,9329 & 0,9731 \\
Índice de peróxido & 0,8274 & 0,9728 & 0,9163 \\
& & $\mathbf{R}^{2} \mathbf{c o n} \mathbf{0 , 3} \%$ \\
Ácidos grasos libres (\%) & 0,8494 & 0,9598 & 0,8332 \\
Índice de peróxido & 0,8449 & 0,8597 & 0,7854 \\
\hline
\end{tabular}

Elaboración propia

Mediante extrapolación se obtuvo la velocidad de reacción (k) a cada dosis de aceite esencial de orégano y a una temperatura de $20^{\circ} \mathrm{C}$ (tabla 4). Como se puede observar, la velocidad de reacción es mayor cuando la aplicación de aceite esencial es menor. 
Tabla 4

Velocidad de reacción ( $k$ ) para una dosis de 0,1 \% y 0,3\% de aceite esencial

\begin{tabular}{lcc}
\hline \multirow{2}{*}{ Factor de calidad } & \multicolumn{2}{c}{$\mathbf{k}\left(\right.$ días $\left.^{-1}\right)$} \\
\cline { 2 - 3 } & $\mathbf{0 , 1} \%$ & $\mathbf{0 , 3} \%$ \\
\hline Acidez (\%) & 0,0160 & 0,0080 \\
Índice de peróxidos & 0,5103 & 0,3634 \\
\hline
\end{tabular}

Elaboración propia

Los valores de vida en anaquel obtenidos en cuanto al porcentaje de acidez e índice de peróxido (tabla 5) reportaron que a una dosis de $0,3 \%$ de aceite esencial se logra que el producto incremente su tiempo de vida útil en comparación con las muestras que contenían $0,1 \%$ de aceite esencial.

Tabla 5

Vida en anaquel del aceite de oliva extravirgen con dosis de 0,1\% y 0,3\% de aceite esencial de orégano

\begin{tabular}{lcc}
\hline \multicolumn{1}{c}{ Factor de calidad } & $\mathbf{0 , 1} \%$ & $\mathbf{0 , 3} \%$ \\
\hline Acidez $\%$ & 39,5 días & 75,6 días \\
Índice de peróxidos & 20,6 días & 28,9 días \\
\hline
\end{tabular}

Elaboración propia

\section{CONCLUSIONES}

- $\mathrm{Al}$ aumentar la temperatura de almacenamiento se incrementa la oxidación y la producción de ácidos grasos libres en el aceite de oliva extravirgen.

- El aceite de oliva extravirgen almacenado a temperaturas de $50{ }^{\circ} \mathrm{C}$, $60^{\circ} \mathrm{C}$ y $70^{\circ} \mathrm{C}$ presentó menores cambios en el \% de acidez e índice de peróxidos cuando la dosis de aceite esencial de orégano fue de $0,3 \%$.

- En el aceite de oliva extravirgen estudiado se estimó que la vida en anaquel en función de la acidez es de 39,5 días y de 75,6 días a una dosis de aplicación de 0,1\% y 0,3\% de aceite esencial de orégano, respectivamente. 
- La vida en anaquel en función del índice de peróxido fue de 20,6 días y de 28,9 días a una dosis de aplicación de $0,1 \%$ y 0,3 \% de aceite esencial de orégano, respectivamente.

\section{REFERENCIAS}

Acevedo, D., Navarro, M., y Monroy, L. (2013). Composición química del aceite esencial de hojas de orégano (Origanum vulgare). Información Tecnológica, 24(4), 43-48.

Amadio, C., Medina, R., Dediol, C., Zimmerman, M., y Miralles, S. (2011). Aceite esencial de orégano: un potencial aditivo alimentario. Revista de la Facultad de Ciencias Agrarias Uncuyo, 43, 237-245.

Aparicio, R., y Harwood, J. (2003). Manual del aceite de oliva. Madrid: Antonio Madrid Vicente Ediciones.

Asencio, C. (2013). Utilización de aceites esenciales de variedades de orégano como conservante antimicrobiano, antioxidante y de las propiedades sensoriales de alimentos: quesos cottage, ricota $y$ aceite de oliva. (Tesis para optar el grado de doctor en Ciencias Agropecuarias). Universidad Nacional de Córdoba. Córdoba, Argentina.

Cervato, G., Carabelli, M., Gervasio, S., Cittera, A., Cazzola, R., y Cestaro, B. (2000). Antioxidant properties of oregano (Origanum vulgare) leaf extracts. Journal of Food Biochemistry, 24(6), 453-465.

Chaquilla, G., Torres, V., Ballinas, M., Gastélum, M., Silva, R., y Nevárez-Moorillón, G. (2008). Actividad antioxidante del aceite esencial de orégano mexicano (lippia berlandieri schauer) en sistemas alimenticios. Respyn (edición especial), 1, 14-15.

Consejo Oleícola Internacional (2013). Norma comercial aplicable a los aceites de oliva y los aceites de orujo de oliva. COI, 15(3).

Gutiérrez, M. (2008). Caracterización e identificación de componentes del aceite esencial de cedrón (Aloysia triphylla). (Tesis para optar el grado de Magister Scientiae). Universidad Nacional Agraria La Molina. Lima, Perú.

Labuza, T., Riboh, D. (1982). Theory and application of Arrhenius kinetics to prediction of nutrient losses in food. Food Technology, 36, 66 . 
Lawson, H. (1999). Aceites y grasas alimentarias. Zaragoza, España: Acribia Editorial.

Morelló, J., Motilva, M., Tovar, M., y Romero, M. (2004). Changes in commercial virgin olive oil (cv Arbequina) during storage, with special emphasis on the phenolic fraction. Food Chemistry, 85(3), 357-364.

Soler, A. (2009). Estudio de la capacidad antioxidante y la biodisponibilidad de los compuestos fenólicos del aceite de oliva. Primeras etapas en el desarrollo de un aceite de oliva funcional. (Tesis para optar el grado de doctor). Universitat de Lleida. Lleida, España. 
